Bull. Austral. Math. Soc.

20D10, 20F 17

VOL. 49 (1994) [325-332]

\title{
EXAMPLES OF SUPERSOLUBLE LOCKETT SECTIONS
}

\section{MaRTin MENTh}

Two Lockett sections of Fitting classes of supersoluble groups are determined. One of these sections has only one member, a minimal supersoluble nonnilpotent Fitting class.

\section{INTRODUCTION}

Recently a supersoluble Fitting class $\mathfrak{U}$ was presented [6]. This is a construction similar to that of Hawkes in [3], which yields metanilpotent Lockett classes, a fact proved by Brison [2]. The existence of supersoluble Lockett classes is assured by the following

THEOREM 1.1. Let $\mathfrak{X}$ be a subgroup and quotient group closed class of groups and $\mathfrak{F}$ be a Fitting class with $\mathfrak{F} \subseteq \mathfrak{X}$. Then $\mathfrak{F}^{*} \subseteq \mathfrak{X}$. Therefore every supersoluble Fitting class $\mathfrak{F}$ has a supersoluble Lockett section $\left[\mathfrak{F}_{*}, \mathfrak{F}^{*}\right]$.

Proof: $\mathfrak{F}^{*}=\left\{G \in \mathfrak{S} \mid(G \times G)_{\mathfrak{F}}=\left(G_{\mathfrak{F}} \times G_{\mathfrak{F}}\right)\left\langle\left(g^{-1}, g\right) \mid g \in G\right\rangle\right\}$ due to [5].

$\left.D:=\left\langle\left(g^{-1}, g\right)\right| g \in G\right)$ is a subgroup of $(G \times G)_{\mathfrak{F}} \in \mathfrak{X}$ and therefore $D \in \mathfrak{X}$. The projection $\pi$ from $D$ onto its second component is an epimorphism from $D$ onto $G$. Hence $G \cong D / \operatorname{Ker}(\pi) \in \mathfrak{X}$.

In this paper we obtain two Lockett sections of Fitting subclasses of $\mathfrak{U}$, the one of $\mathfrak{U}$ itself and the Lockett section of a minimal nonnilpotent subclass $\mathfrak{W}$ of $\mathfrak{U}$. $\mathfrak{U}$ turns out to be a Lockett class but not to coincide with its lower star, whereas the Lockett section of $\mathfrak{W}$ has only one member.

All groups will be finite and soluble. $\mathfrak{F}(G)$ denotes the Fitting class generated by $G$. The notations and properties of Lockett's "star"-operators can be found in [5].

\section{A LOCKETT SECTION WITH TWO MEMBERS}

Notation. Let $p$ be prime, $p \equiv 1 \bmod 3, n$ a primitive 3 rd unit root in $G F(p)$.

$$
\begin{aligned}
& T:=\left\langle a, b \mid a^{p}=b^{p}=[a, b, a, a]=[a, b, a, b]=[a, b, b, b]=1\right\rangle \\
& U:=\left\langle T, s \mid s^{3}=1, a^{*}=a^{n}, b^{*}=b^{n}\right\rangle
\end{aligned}
$$

Received 27th May, 1993.

This article arises from the author's dissertation written at the University of Würzburg in 1992 under the direction of Professor Dr. H. Heineken.

Copyright Clearance Centre, Inc. Serial-fee code: 0004-9729/84 \$A2.00+0.00. 
Then $T$ is free with respect to the properties (nilpotent of class three, exponent $p$, two generators), $|T|=p^{5}$ and $Z(U)=Z(T) \cong C_{p} \times C_{p}$.

$G$ is called a central product of normal subgroups $G_{1}, \ldots, G_{m}$ if $G=G_{1} \cdot \ldots \cdot G_{m}$ and $\left[G_{i}, G_{j}\right]=1$ for $i \neq j$.

Let now $\mathfrak{U}_{0}$ be the class of all finite groups $G=X Y$ with

(i) $X:=O_{p}(G)$ is a central product of groups $T_{i} \cong T$ (the empty product, that is, $O_{p}(G)=1$, being admitted).

(ii) $Y \in S y l_{3}(G), \forall i:\left[Y / C_{Y}\left(T_{i}\right) \cong C_{3}\right.$ and $\left.T_{i} \rtimes\left(Y / C_{Y}\left(T_{i}\right)\right) \cong U\right]$.

Further let

$$
\begin{aligned}
G \in \mathfrak{U}: & \Longleftrightarrow G=O_{p}(G) \cdot O^{p}(G) \text { and } O^{p}(G) \in \mathfrak{U}_{0} \\
& \Longleftrightarrow G \in \mathfrak{S}_{p} \mathfrak{S}_{3} \text { and } O^{p}(G) \in \mathfrak{U}_{0},
\end{aligned}
$$

where $O^{p}(G)$ is the largest $p$-perfect normal subgroup of $G$. Then $\mathfrak{U}=\mathfrak{F}(U)$, and $\mathfrak{U}$ is supersoluble according to $[6,(4.1)$ and (4.2)].

LEMMA 2.1.

(a) Let $G \in \mathfrak{S}_{p} \mathfrak{S}_{3}$. Then $G \in \mathfrak{U} \Longleftrightarrow G / O_{3}(G) \in \mathfrak{U}$.

(b) $\mathfrak{U}=\mathfrak{H}:=\left\{G \in \mathfrak{S}_{p} \mathfrak{S}_{3} \mid O^{p}\left(G / O_{3}(G)\right) \in \mathfrak{U}_{0}\right\}$.

Proof: Set $\bar{G}:=G / O_{3}(G)$. Since $O_{p}\left(O^{p}(G)\right) \cong O_{p}\left(O^{p}(G)\right)$, we have to check only the operations induced on the central factors of $O_{p}\left(O^{p}(G)\right)$ and $O_{p}\left(O^{p}(G)\right)$ by the 3-elements of $G$ and $\bar{G}$. By assumption $G \in \mathfrak{S}_{p} \mathfrak{S}_{3}$, so $O_{3}(G)$ centralises all pelements of $G$ and the assertion (a) is clear. Let $G \in \mathfrak{H} . G / O_{3}(G)=O_{p}\left(G / O_{3}(G)\right)$. $O^{p}\left(G / O_{3}(G)\right)$ and $O^{p}\left(G / O_{3}(G)\right) \in \mathfrak{U}_{0}$, so $G / O_{\mathfrak{s}}(G) \in \mathfrak{U}$ and therefore $G \in \mathfrak{U}$. The converse direction is clear because of (a).

Part (b) of the preceeding lemma shows that the class $\mathfrak{U}$ has a structure similar to that of the metanilpotent Fitting classes constructed by Hawkes [3], although item $d$ of hypothesis 5.1 is not fulfilled. In fact $\mathfrak{U}=\mathfrak{F}\left(3^{\prime}, p^{\prime}, \mathfrak{U}_{0}\right) \cap \mathfrak{S}_{p} \mathfrak{S}_{3}$ in the notation of Hawkes. Now we can use Brison's proof almost verbatim to show that the supersoluble Fitting class $\mathfrak{U}$ is a Lockett class, too. In order to avoid the rewriting, the reader is referred to [3] and [2] for the necessary parts of the cited proofs.

Lemma 2.2. $\mathfrak{U}$ is a Lockett class.

PrOOF: Using the notation of [3] and [2] it remains only to show that $C_{S}\left(Q_{j}\right) \cong C_{3}$ and $S / C_{S}\left(Q_{j}\right)$ operates on $Q_{j} / Q_{j}^{\prime}$ by a power automorphism. Let $j \in \mathcal{S}_{i}$. It is already known that $S=A C_{i}$ and $\left[Q_{j}, C_{i}\right] \leqslant\left[F_{i}, C_{i}\right]=1$, so $C_{S}\left(Q_{j}\right) \geqslant C_{i}$. Let $y:=\left(g^{-1}, g\right)$ be the generating element of $A$. By hypothesis $y$ raises the elements of $P_{j} / P_{j}^{\prime}=P_{j} / Q_{j}^{\prime}$ to the power of $n$. Let $\{a, b\}$ be a generating system of $Q_{j}$. Since $Q_{j} \leqslant P_{j} H_{i}$, there are elements $a^{\prime}, b^{\prime} \in P_{j}$ and $h_{1}, h_{2} \in H_{i}$ with $a=a^{\prime} h_{1}, b=b^{\prime} h_{2} . H_{i} \leqslant K^{\prime}$, hence 
$a^{\prime}$ and $b^{\prime}$ are not elements of $P_{j}^{\prime}$. Therefore we have $z_{1}, z_{2} \in K^{\prime}$ with $\left(a^{\prime}\right)^{y}=\left(a^{\prime}\right)^{n} z_{1}$ and $\left(b^{\prime}\right)^{y}=\left(b^{\prime}\right)^{n} z_{2}$. Now $a^{y}=\left(a^{\prime}\right)^{y} h_{1}^{y}=\left(a^{\prime}\right)^{n} z_{1} h_{1}^{y}$ and $z_{1} h_{1}^{y} \in K^{\prime} \cap Q_{j}=Q_{j}^{\prime}$. That means: $y$ raises $a$ modulo $Q_{j}^{\prime}$ to the $n$-th power. The same holds for $b$. Moreover $|S|=3^{2}$ and $|A|=3$ so $C_{i}=C_{S}\left(Q_{j}\right)$ and $S / C_{i} \cong C_{3}$.

Theorem 2.3. Blessenohl and Gaschütz [1, Satz 3.3]. Let $p$ be a prime, $S$ a subgroup of the multiplicative subgroup $G F(p)^{*}$ of $G F(p)$. Let $G$ be a soluble group, $g \in G$. Fix a principal series of $G$ and denote by $\mathcal{P}$ the set of the $p$-factors of this series. Then there is (uniquely determined up to similarity) a matrix $M(g ; H / K)$, that describes the operation of $g$ on the $p$-chief factor $H / K$. Define further a mapping $w_{G}: G \longrightarrow G F(p)^{*}$ by

$$
g \longmapsto \prod_{H / K \in \mathcal{P}} \operatorname{det}(M(g ; H / K))
$$

Then $\mathfrak{G}_{p}(S):=\left\{G \in \mathfrak{S} \mid \forall g \in G: w_{G}(g) \in S\right\}$ is a normal Fitting class.

Definition 2.4: $\mathfrak{V}:=\mathfrak{U} \cap \mathfrak{G}_{p}(1) ; \quad G_{m}:=\left(T_{1} \times \ldots \times T_{m}\right) \rtimes\langle s\rangle$, where all the $T_{i}$ are normal in $G_{m}$ and isomorphic to $T,\langle s\rangle \cong C_{3}$, and $s$ raises the elements of $T_{i} / T_{i}^{\prime}$ to the $n$-th power.

Since $\mathfrak{V}$ contains the nonnilpotent group $G_{3}$ but not the group $U, \mathfrak{V}$ lies strictly between $\mathfrak{S}_{p} \mathfrak{S}_{3} \cap \mathfrak{N}$ and $\mathfrak{U}$. Moreover it is the lower bound of the Lockett section of $\mathfrak{U}$ (Lemma 2.5).

In the following proofs automorphisms $\alpha$ are considered which raise the elements of the commutator factor group $T_{i} / T_{i}^{\prime}$ of a central product $T_{1} \cdot \ldots \cdot T_{m}$ to some power $n^{\lambda_{i}}$. Such automorphisms will be denoted briefly by $\left(n^{\lambda_{1}}, \ldots, n^{\lambda_{m}}\right)$.

LEMMA 2.5. $\mathfrak{V}=\mathfrak{F}\left(G_{3}\right)=\mathfrak{U}_{*}$

PROOF:

(i) We have to show that all groups $G \in \mathfrak{U}$ with "trivial determinant" are contained in $\mathfrak{F}\left(G_{3}\right)$. Because $G=O_{p}(G) \cdot O^{p}(G)$, it is sufficient to prove that the p-perfect $\mathfrak{V}$ groups are in $\mathfrak{F}\left(G_{3}\right)$. Moreover the problem can be reduced to the following case: $G=X\langle s\rangle, X=T_{1} \cdot \ldots \cdot T_{m}$ is a central product of $T_{i} \cong T,\langle s\rangle \cong C_{3}$, and $s$ operates on $X$ as $\left(n^{\lambda_{1}}, \ldots, n^{\lambda_{m}}\right)$ with $\lambda_{i} \neq 0$.

(ii) Set $W:=\left(T_{1} \times T_{2}\right) \times\langle s\rangle$, where $s$ acts as $\left(n, n^{2}\right)$. In order to prove $W \in \mathfrak{F}\left(G_{3}\right)$, we consider the extension of a direct product $P:=T_{1} \times T_{2} \times T_{3} \times T_{4}$ of four copies of $T$ by automorphisms $\alpha=(n, n, n, 1)$ and $\beta=\left(n^{2}, n^{2}, 1, n^{2}\right) .\langle P, \alpha, \beta\rangle$ is a normal product of $\left\langle T_{1}, T_{2}, T_{3}, \alpha\right\rangle \cong G_{3}$ and $\left\langle T_{1}, T_{2}, T_{4}, \beta\right\rangle \cong G_{3}$ and possesses a subnormal subgroup $\left\langle T_{3}, T_{4}, \alpha \beta\right\rangle \cong W$.

(ii) Now (ii) will be generalised to groups $H:=\left(T_{1} \cdot T_{2}\right) \rtimes\langle s\rangle$, where $T_{1} \cdot T_{2}$ is a central product of the $T_{i}$ : Take a third copy $T_{3}$ of $T$ and form the direct product $P:=\left(T_{1} \cdot T_{2}\right) \times T_{3}$. Then there exist automorphisms $\alpha=\left(n, 1, n^{2}\right)$ and $\beta=\left(1, n^{2}, n\right)$. 
$\langle P, \alpha, \beta\rangle$ is a normal product of $\left\langle T_{1}, T_{3}, \alpha\right\rangle \cong W$ and $\left\langle T_{2}, T_{3}, \beta\right\rangle \cong W$, so it is contained in $\mathfrak{F}\left(G_{3}\right)$ by (ii). Moreover $\left\langle T_{1}, T_{2}, \alpha \beta\right\rangle$ is a subnormal subgroup of $\langle P, \alpha, \beta\rangle$ and isomorphic to $H$. Hence $H \in \mathfrak{F}\left(G_{3}\right)$.

(iv) Using the same method as in (iii) one obtains $H:=\left(T_{1} \cdot T_{2} \cdot T_{3}\right) \rtimes\langle s\rangle \in \mathfrak{F}\left(G_{3}\right)$, where $T_{1} \cdot T_{2} \cdot T_{3}$ is a central product and all factors $T_{i} / T_{i}^{\prime}$ are raised to the $n$-th power by $s$.

(v) In the general case the group $G$ can be built by the prototypes constructed in (iii) and (iv): Imagine the factors $T_{i}$ numbered in such a way that $s$ raises $T_{1}, \ldots, T_{k}$ modulo the respective commutator subgroups to the power of $n$ and $T_{k+1}, \ldots, T_{m}$ to the power of $n^{2}$. Without loss of generality $k \geqslant 1$. Setting $l:=m-k$ one obtains $w_{G}(s)=n^{k+2 l} \equiv 1 \bmod 3$; that means $k \equiv l \bmod 3$. There are integers $\kappa, \lambda$ and $\mu$ with $k=3 \kappa+\mu, l=3 \lambda+\mu$. Hence $G$ is isomorphic to a normal product of $\kappa+\lambda$ groups of type (iv) and $\mu$ groups of type (iii).

(vi) $\mathfrak{U}=\mathfrak{F}(U), \exp \left(U / U^{\prime}\right)=3$ and $G_{3}$ is isomorphic to the group $U(3)$ defined in [4]. According to $[4$, Satz $1.3, \mathrm{~b}]$ we get therefore $\mathfrak{U}_{*}=\mathfrak{F}\left(G_{3}\right)=\mathfrak{V}$.

LEMMA 2.6. Let $G \in \mathfrak{U}, O_{p}\left(O^{p}(G)\right)$ be a direct product of copies $T_{i}$ of $T$. $G \in \mathfrak{U} \backslash \mathfrak{V} \Longleftrightarrow \mathfrak{F}(G)=\mathfrak{U}$ and $G \in \mathfrak{V} \backslash \mathfrak{N} \Longleftrightarrow \mathfrak{F}(G)=\mathfrak{V}$. Therefore $\mathfrak{F}\left(G_{m}\right)=\mathfrak{U}$ if $m \not \equiv 0 \bmod 3$, and $\mathfrak{F}\left(G_{m}\right)=\mathfrak{V}$ if $m \equiv 0 \bmod 3$.

Proof: $G \in \mathfrak{U} \backslash \mathfrak{V}\left(\mathfrak{V}\right.$ respectively) if and only if $O^{p}(G) \in \mathfrak{U} \backslash \mathfrak{V}(\mathfrak{V}$ respectively). Hence $G$ can be reduced to the following form: $G:=X \rtimes\langle s\rangle, X=T_{1} \times \ldots \times T_{m}$ all $T_{i} \triangleleft$ $G$ and $T_{i} \cong T,\langle s\rangle \cong C_{3}, s$ acts on $X$ like $\left(n, \ldots, n, n^{2}, \ldots, n^{2}\right)$, the number of entries " $n$ " being $k$. Set $l:=m-k$ and assume without loss of generality $1 \leqslant l \leqslant k$. $G \in \mathfrak{V} \Longleftrightarrow w_{G}(s)=1 \Longleftrightarrow k-l \equiv 0 \bmod 3 . \mathfrak{F}(G)$ also contains the extension of $X$ by $\alpha:=(\underbrace{n^{2}, \ldots, n^{2}}_{l}, \underbrace{n, \ldots, n}_{k})$ and $\beta:=(n, \underbrace{n^{2}, \ldots, n^{2}}_{l-1}, \underbrace{n, \ldots, n}_{k-1}, n^{2})$ and therefore the extension of $T_{1} \times T_{2}$ by $\left(n, n^{2}\right)$. Anyway $G_{3}$ can be found in $\mathfrak{F}(G)$ and subsequently we get $\mathfrak{V} \subseteq \mathfrak{F}(G)$. If $G \notin \mathfrak{V}$ then $k-l \not \equiv 0 \bmod 3$ and $\mathfrak{F}(G)$ contains a group isomorphic to $G_{k-1}$ and then the group $G_{1} \cong U$ or the group $G_{2}$. Since $G_{3} \in \mathfrak{F}(G), G_{2} \in \mathfrak{F}(G)$ implies $G_{1} \in \mathfrak{F}(G)$ and finally $\mathfrak{F}(G)=\mathfrak{U}$.

LemMa 2.7. Let $T_{1} \cdot T_{2}$ be a central product of two copies of $T$ and $G:=$ $\left(T_{1} T_{2}\right) \rtimes\langle s\rangle$ with $\langle s\rangle \cong C_{3}$, where $s$ raises the elements of $T_{i} / T_{i}^{\prime}$ to the power of $n^{\lambda_{i}}$.

(a) If $\lambda_{1}=\lambda_{2} \neq 0$, then $\mathfrak{F}(G)=\mathfrak{U}$.

(b) If $\lambda_{1}=1, \lambda_{2}=2$ and $\left|T_{1} \cap T_{2}\right|=p$, then $\mathfrak{F}(G)=\mathfrak{V}$.

Proof:

(a) We can assume $\lambda_{1}=1$. The case $T_{1} \cap T_{2}=1$ is done in (2.6), so there remain two cases:

CASE 1. $\left|T_{1} \cap T_{2}\right|=p$. Let $X$ be a central product of four copies of $T$ with the fol- 
lowing intersections: $\left|T_{1} \cap T_{2}\right|=\left|T_{2} \cap T_{3}\right|=\left|T_{3} \cap T_{4}\right|=p ; \quad T_{1} \cap T_{4}=1 ; \quad\left(T_{1} \cap T_{2}\right) \times$ $\left(T_{2} \cap T_{3}\right)=Z\left(T_{2}\right) ; \quad\left(T_{2} \cap T_{3}\right) \times\left(T_{3} \cap T_{4}\right)=Z\left(T_{3}\right)$. Then $\mathfrak{F}(G)$ contains the extensions $H_{1}:=\langle X, \alpha\rangle, H_{2}:=\langle X, \beta\rangle$ and $H_{3}:=\langle X, \gamma\rangle$ with $\alpha:=(n, n, 1,1), \beta:=(1,1, n, n)$ and $\gamma:=\left(1, n^{2}, n^{2}, 1\right) . \alpha \beta \gamma=(n, 1,1, n)$, and $H:=\langle X, \alpha \beta \gamma\rangle$ possesses a normal subgroup $N \cong G_{2}$. According to $(2.6)$ we get $\mathfrak{F}(G)=\mathfrak{U}$.

CASE 2. $\left|T_{1} \cap T_{2}\right|=p^{2}$. Let again $X$ be a central product of four copies of $T$, but now $Z\left(T_{1}\right)=T_{i} \cap T_{j}$ for all $i, j \in\{1, \ldots, 4\} . \mathfrak{F}(G)$ contains the extensions of $X$ by $\alpha:=(n, n, 1,1), \quad \beta:=\left(1,1, n^{2}, n^{2}\right), \quad \gamma:=(n, 1,1, n)$ and $\varepsilon:=(n, 1, n, 1)$, and therefore with $\alpha \beta \gamma \varepsilon=(1, n, 1,1)$ and at last a group isomorphic to $U$.

(b) There exists a central product $P=T_{1} \cdot T_{2} \cdot T_{3}$ of three copies of $T$ with $T_{2} \cdot T_{3} \cong$ $T_{1} \cdot T_{2}$ and $T_{1} \cap T_{3}=1$. Set $\sigma:=\left(n, n^{2}, 1\right)$ and $\tau:=\left(1, n, n^{2}\right)$. Hence $\langle P, \sigma, \tau\rangle$ is a normal product of two copies of $G$ and has a subnormal subgroup $N=\left\langle T_{1} \times T_{3}, \sigma \tau\right\rangle$ with $\mathfrak{F}(N)=\mathfrak{V}$ according to (2.6).

Finally the Lockett section of $\mathfrak{U}$ is found, and it turns out that $\mathfrak{U}$ fulfills the Lockett conjecture:

THEOREM 2.8. The Lockett section of $\mathfrak{U}$ is $\{\mathfrak{V}, \mathfrak{U}\}$.

Proof: Let $\mathfrak{X}$ be a Fitting class with $\mathfrak{U}_{*}=\mathfrak{V} \subseteq \mathfrak{X} \subseteq \mathfrak{U}$. $\mathfrak{X}$ contains a group $G \in \mathfrak{U}_{0} \backslash \mathfrak{V}$ constructed analogously to the group $G$ in the proof of (2.6) with the only alteration that $X=T_{1} \cdot \ldots \cdot T_{m}$ is now a central (eventually not a direct) product. Define $k, m, l$ as in (2.6). Now $k-l \not \equiv 0 \bmod 3$. Let further $\alpha_{j}$ be the automorphism of $X$ which centralises $T_{1}, \ldots, T_{j}$ and operates on the remaining factors $T_{i}$ in the same way as $s$. One obtains $X \rtimes\left\langle\alpha_{1}\right\rangle \in \mathfrak{V}$ if $k-l \equiv 1 \bmod 3$, and $X \rtimes\left\langle\alpha_{2}\right\rangle \in \mathfrak{V}$ if $k-l \equiv 2 \bmod 3$. In the first case $X \times\left\langle s \alpha_{1}^{-1}\right\rangle \in \mathfrak{X}$ has a normal subgroup isomorphic to $U$, and $\mathfrak{X}=\mathfrak{U}$. In the second case $X \times\left\langle s \alpha_{2}^{-1}\right\rangle \in \mathfrak{X}$ has a normal subgroup that fulfills the hypothesis of (2.7), and again $\mathfrak{X}=\mathfrak{U}$.

\section{A LOCKETT SECTION WITH ONE MEMBER}

The Fitting class $\mathfrak{V}$ arose from intersecting $\mathfrak{U}$ with the normal Fitting class $\mathfrak{G}_{p}(1)$. Since all intersections of $\mathfrak{U}$ with normal Fitting classes will appear in the Lockett section of $\mathfrak{U}$, there is no hope for finding more subclasses of $\mathfrak{U}$ by constructing such intersections. However there is at least one other nonnilpotent subclass of $\mathfrak{U}$, and that class coincides with its upper and lower star. The Fitting classes generated by the groups $G_{m}$ can be determined easily because the central factors $T_{i}$ of $O_{p}\left(G_{m}\right)$ intersect trivially. A similar situation arises when these intersections are as great as possible.

Definition 3.1:

(a) Let $H_{m}:=\left(T_{1} \cdot \ldots \cdot T_{m}\right) \rtimes\langle s\rangle$ fulfill the following hypothesis: 
(i) $T_{1} \cdot \ldots \cdot T_{m}$ is a central product of normal subgroups $T_{i} \cong T$ of $H_{m}$.

(ii) For all $i, j \in\{1, \ldots, m\}: T_{i} \cap T_{j}=Z\left(T_{i}\right)=Z\left(T_{j}\right)$.

(iii) $\langle s\rangle \cong C_{3}$ and $s$ raises the elements of $T_{i} / T_{i}^{\prime}$ to the power of $n$.

(b) Let $K:=\left(T_{1} \cdot T_{2}\right) \times\langle s\rangle$ fulfill hypotheses (i) and (ii) of (a), $\langle s\rangle \cong C_{3}$ and $s$ operating on $T_{1} T_{2}$ as $\left(n, n^{2}\right)$.

Analogously to (2.6) one easily obtains:

Lemma 3.2. $\mathfrak{F}\left(H_{m}\right)=\mathfrak{U}$ if $m \not \equiv 0 \bmod 3$ and $\mathfrak{F}\left(H_{m}\right)=\mathfrak{F}\left(H_{3}\right)=\mathfrak{F}(K)$ if $m \equiv 0 \bmod 3$.

DEFinition 3.3: Let $\mathcal{M}$ be the class of all central products of copies $T_{i}$ of $T$ with $T_{i} \cap T_{j}=Z\left(T_{i}\right)=Z\left(T_{j}\right)$ for all $i$ and $j$ and $\mathfrak{W}$ be the class of all $G \in \mathscr{U}$ such that $O_{p}\left(O^{p}(G)\right)$ is a central product of maximal $\mathcal{M}$-groups $M_{1}, \ldots, M_{m}$ with the additional property that $\left\langle M_{i}, s\right\rangle \in \mathfrak{V}$ for all $i \in\{1, \ldots, m\}$ and all 3-elements $s \in G$.

The case $m=0$, that means $O^{p}(G) \in \mathfrak{S}_{3}$, is admitted. In accordance with [6, 3.5], the factors $M_{i}$ are uniquely determined up to permutation.

Proposition 3.4. $\mathfrak{W}$ is a Fitting class, and $\mathfrak{W}=\mathfrak{F}\left(H_{3}\right) \subsetneq \mathfrak{V}$.

Proof:

(i) Let $G_{1} \cdot G_{2}$ be a normal product of groups $G_{i} \in \mathfrak{W}$. Without loss of generality we can assume $G_{1}, G_{2} \in \mathfrak{U}_{0}$. If at least one of the two factors $G_{i}$ is a 3-group, then $G_{1} G_{2} \in \mathfrak{W}$ is clear. Let be $O_{p}\left(G_{1}\right)=M_{1} \cdot \ldots \cdot M_{m}, \quad O_{p}\left(G_{2}\right)=L_{1} \cdot \ldots \cdot L_{l}, m, l \geqslant 1$, as in (3.3).

(1) For all $i \in\{1, \ldots, m\}, j \in\{1, \ldots, l\}$, either $M_{i} L_{j}$ is a maximal $\mathcal{M}$ normal subgroup of $G_{1} G_{2}$, or $M_{i}$ and $L_{j}$ are situated in different maximal $\mathcal{M}$-normal subgroups of $G_{1} G_{2}$. The first case arises if and only if in the decomposition of $L_{j}$ into its factors $T_{k} \cong T$ there is a factor $T_{k_{0}}$ with $T_{k_{0}} \cap$ $M_{i}=Z\left(M_{i}\right)$. Then this equation is valid also for all the other factors $T_{k_{i}}$ of $L_{j}$, because $L_{j} \in \mathcal{M}$.

(2) By (1) $O_{p}\left(G_{1} G_{2}\right)$ is a central product of maximal $\mathcal{M}$-groups $K_{1}, \ldots, K_{k}$, where $K_{i}$ satisfies one of the following conditions:

(I) $K_{i}=M_{i}$ and $K_{i} \cap G_{2} \subseteq Z\left(G_{2}\right)$,

(II) there is a $j \in\{1, \ldots, l\}$ with $K_{i}=L_{j}$ and $K_{i} \cap G_{1} \subseteq Z\left(G_{1}\right)$,

(III) there is a $j \in\{1, \ldots, l\}$ with $K_{i}=M_{i} L_{j}$.

(3) It remains to check the determinant of the operation induced by a 3-element $s \in G_{1} G_{2}$ on $K_{i}$. Since $Y \in S y l_{3}\left(G_{1} G_{2}\right)$ is a product of $Y_{1}$ and $Y_{2}$ with $Y_{i} \in S y l_{3}\left(G_{i}\right)$, it suffices to consider $s \in Y_{1}$ and $t \in Y_{2}$. 
CASE I. For $s \in G_{1}$ the assertion is clear. According to $[6,3.7 . c], t \in G_{2}$ centralises $K_{i}$.

CASE II. This case is analogous to case I.

CASE III. Let $L_{j}=T_{1} \cdot \ldots \cdot T_{r}$ be the decomposition of $L_{j}$, uniquely up to permutation. Again in consequence of $[6,3.7 . c]$, a factor $T_{\nu}$ is either identical to some central factor of $M_{i}$ or it is centralised by $s \in G_{1}$. Hence $\left\langle M_{i} L_{j}, s\right\rangle$ and $\left\langle M_{i} L_{j}, t\right\rangle$ are contained in $\mathfrak{V}$.

(ii) Let be $G \in \mathfrak{W}$ and let $N$ be a normal subgroup of $G . O_{p}\left(O^{p}(N)\right)$ is a central product of normal subgroups $T_{1}, \ldots, T_{m}$, isomorphic to $T$, and $\left\{T_{1}, \ldots, T_{m}\right\}$ is a subset of the set of the central factors $T_{i}$ of $O_{p}\left(O^{p}(G)\right)$. Fix a maximal $\mathcal{M}$-factor $M_{i}$ of $O_{p}\left(O^{p}(G)\right)$. Passing from $G$ to $N$, due to $\left[6,3.8\right.$.a] only factors $T_{j} \leqslant M_{i}$ that are centralised by all 3-elements $s \in N$ can disappear. So $M_{i} \cap N$ is a $\mathcal{M}$-group with $\left\langle M_{i} \cap N, s\right\rangle \in \mathfrak{D}$ for all 3-elements $s \in N$. Moreover $M_{i} \cap N$ is a maximal $\mathcal{M}$-factor of $N$. Since $O_{p}\left(O^{p}(N)\right)=\left(M_{1} \cap N\right) \cdot \ldots\left(M_{m} \cap N\right)$, we get $N \in \mathfrak{W}$.

(iii) $H_{3} \in \mathfrak{W}$, and $G_{3} \notin \mathfrak{W}$. Therefore $\mathfrak{N} \subsetneq \mathfrak{F}\left(H_{3}\right) \subseteq \mathfrak{W} \subsetneq \mathfrak{V}$. Suppose now $M \in$ $\mathcal{M}$ and $\langle s\rangle \cong C_{3}$ with $\langle M, s\rangle \in \mathfrak{V}$. Since $\mathfrak{F}\left(H_{3}\right)=\mathfrak{F}(K)$, one gets $\langle M, s\rangle \in \mathfrak{F}\left(H_{3}\right)$ using the same method as in part (v) of (2.5). Analogously to [6, 3.3] this leads to $\mathfrak{W} \subseteq \mathfrak{F}\left(\boldsymbol{H}_{3}\right)$.

Proposition 3.5. $\mathfrak{W}$ is the only minimal nonnilpotent Fitting subclass of $\mathfrak{U}$.

Proof: We fix $G \in \mathfrak{U} \backslash \mathfrak{N}$ and show $\mathfrak{W} \subseteq \mathfrak{F}(G)$. Let $G$ fulfill the usual hypothesis (see (2.6)), the operation of $s$ being described by $\left(n^{\lambda_{1}}, \ldots, n^{\lambda_{m}}\right), \lambda_{i} \neq 0$. Let $F$ be free with respect to the properties (nilpotent of class 3 , exponent $p$, $2(m+1)$ generators). It is possible to find a normal subgroup $N \leqslant Z(F)$ such that $P:=F / N$ is a central product of copies $T_{1}, \ldots, T_{m}, T_{m+1}$ of $T$ obeying the condition $T_{1} \cdot \ldots \cdot T_{m} \cong T_{2} \cdot \ldots \cdot T_{m+1} \cong O_{p}(G)$. Now take $\sigma, \tau \in A u t(P)$ defined by $\sigma=\left(n^{\lambda_{1}}, \ldots, n^{\lambda_{m}}, 1\right)$ and $\tau=\left(1, n^{-\lambda_{2}}, \ldots, n^{-\lambda_{m}}, n^{-\lambda_{1}}\right)$. Hence $\langle P, \sigma, \tau\rangle$ is a normal product of two copies of $G$ and has a subnormal subgroup $L=\left\langle T_{1} \cdot T_{m+1}, \sigma \tau\right\rangle$, where $\sigma \tau$ operates as $\left(n^{\lambda_{1}}, n^{-\lambda_{1}}\right)$. If $\left|T_{1} \cap T_{m+1}\right|=p^{2}$, then $L \cong K$ and $\mathfrak{F}(L)=\mathfrak{W}$. Otherwise $\mathfrak{F}(L)=\mathfrak{V}$ due to (2.6) and (2.7.b).

THEOREM 3.6. $\mathfrak{W}_{*}=\mathfrak{W}=\mathfrak{W}^{*}$

Proof: $\mathfrak{W}_{*}=\mathfrak{W}$ is clear by (3.5). $\mathfrak{W}=\mathfrak{W}^{*}$ : Since $\mathfrak{W}^{*} \subseteq \mathfrak{U}^{*}=\mathfrak{U}$, it suffices to show that $(G \times H)_{\mathfrak{W}}=G_{\mathfrak{W}} \times H_{\mathfrak{W}}$ for all $G, H \in \mathfrak{U}$. Further $O^{p}(G \times H)=O^{p}(G) \times$ $O^{P}(H)$, so we can assume $G$ and $H$ to be p-perfect. $G, H \in \mathfrak{U}$, so $O_{p}(G)=T_{1} \cdot \ldots \cdot T_{l}$ and $O_{p}(H)=U_{1} \ldots . U_{k}$ are central products of factors isomorphic to $T$ with the usual operation of the 3-elements. Since $G \cap H=1, O_{p}(G \times H)=T_{1} \cdot \ldots \cdot T_{l} \cdot U_{1} \cdot \ldots \cdot U_{k}$ 
is the corresponding decomposition of $O_{p}(G \times H)$. Let $M_{1}, \ldots, M_{m}$ be the maximal $\mathcal{M}$-normal subgroups of $O_{p}(G \times H)$. Each factor $M_{i}$ is contained in $G$ or in $H$. $(G \times H)_{\mathfrak{W}} \geqslant O_{p}(G \times H)$. Moreover $M_{i}$ is either contained in $O^{p}\left((G \times H)_{\mathfrak{W}}\right)$ or is centralised by all 3-elements $s \in(G \times H)_{\mathfrak{w}}$. Each 3-element $s \in G \times H$ is a product of 3-elements $s_{1} \in G$ and $s_{2} \in H . s=s_{1} s_{2}$ is an element of $(G \times H)_{\mathfrak{W}}$ if and only if it induces on every factor $M_{i}$ an automorphism with "determinant" 1 . In this case $s_{1}$ and $s_{2}$ have this property, too: If $M_{i} \leqslant G, s_{2}$ centralises $M_{i}$. If $M_{i} \leqslant H, s_{1}$ centralises $M_{\mathfrak{i}}$. Hence $s_{1} \in G_{\mathfrak{W}}$ and $s_{\mathfrak{2}} \in H_{\mathfrak{W}}$. Together we get $(G \times H)_{\mathfrak{W}}=G_{\mathfrak{W}} \times H_{\mathfrak{W}}$.

\section{REFERENCES}

[1] D. Blessenohl and W. Gaschütz, 'Über normale Schunck- und Fittingklassen', Math. Z. 118 (1970), 1-8.

[2] O. Brison, 'On a Fitting class of Hawkes', J. Algebra 68 (1981), 28-30.

[3] T. Hawkes, 'On metanilpotent Fitting classes', J. Algebra 63 (1980), 459-483.

[4] K. Johnsen and H. Laue, 'Über endlich erzeugte Fittingklassen', Arch. Math. (Basel) 30 (1978), 350-360.

[5] F.P. Lockett, 'The Fitting class $\mathfrak{F}^{*}$ ', Math. Z. 137 (1974), 131-136.

[6] M. Menth, 'A Family of Fitting classes of supersoluble groups', (submitted).

Mathematisches Institut

Universitāt Würzburg

Am Hubland

D-W-8700 (D-97074) Würzburg

Germany 